\title{
The Existence and Uniqueness of Initial-Boundary Value Problems of the Fractional Caputo-Fabrizio Differential Equations
}

\author{
Şuayip Toprakseven ${ }^{1 *}$ \\ ${ }^{1}$ Faculty of Engineering, Department of Computer Science, Artvin Çoruh University, Artvin, 08100, Turkey \\ * Corresponding author
}

\section{Article Info}

Keywords: Caputo-Fabrizio fractional derivative, Existence and uniqueness of fractional differential equations, Laplace transform, Mass spring damper system.

2010 AMS: 26A33, 34A08.

Received: 5 April 2019

Accepted: 24 April 2019

Available online: 28 June 2019

\begin{abstract}
In this paper, the existence and uniqueness problem of the initial and boundary value problems of the linear fractional Caputo-Fabrizio differential equation of order $\sigma \in(1,2]$ have been investigated. By using the Laplace transform of the fractional derivative, the fractional differential equations turn into the classical differential equation of integer order. Also, the existence and uniqueness of nonlinear boundary value problem of the fractional Caputo-Fabrizio differential equation has been proved. An application to mass spring damper system for this new fractional derivative has also been presented in details.
\end{abstract}

\section{Introduction}

The fractional differential calculus has gained much interest by the many researcher in the last decades and it has strong mathematical background and many papers are attributed to the development of it. Among them, we can cite some e.g, [1, 2, 3]. Fractional calculus has been also used for modelling physical phenomena including control systems, mechanics, viscoelasticity [4, 5, 6]. Up to now, several definition of fractional derivative has been proposed. Some frequently used definition of fractional derivative can be given as the Riemann-Lioville, Caputo, Grünwald-Letnikov [7, 8] and conformable fractional derivative [9, 10, 11, 12, 13]. Among them, the Riemann-Lioville definition requires nonlocal initial condition, so it does not reflect physical experiment while the Caputo definition allows to use the classical initial condition. In the recent years, a new definition of fractional order derivative has been defined by Caputo and Fabrizio [14] with a regular kernel. This new definition can be able to describe better heterogeneousness and systems with different scales with memory effects. The other good property of this new definition is that the real power turn into the integer by the Laplace transformation, thus the exact solution can be easily found for some cases. Some properties of this definition have been studied in [15]. Several papers are devoted to development of this new fractional derivative [16,17]. Some applications based on this new fractional derivative can be found in the papers $[18,19,20,21,22,23]$. In this paper, the previous results will be extended and the existence and uniqueness solution will be given for high order fraction derivative. As an application, a mass-spring-damper system will be analyzed basen on this new derivative. In [15], the results are presented when the fractional order $\alpha \in(0,1)$. The aim of this paper enriches these results for the case when the fractional order of $\alpha+1 \in(1,2)$. In [19], a mass spring damper motion has been studied, but the solution available only for numerical approximation using Laplace transform algorithm. More importantly, they consider the fractional order $2 \alpha \in(1,2)$ when $\alpha \in(0,1)$. However, this is not true when $\alpha \in(0,1 / 2)$. Additionally, the Caputo-Fabrizio fractional operator does not have semigroup property. For this reason, the different cases of the fractional order also have been examined and the exact solution for each case is given for the mass spring damper equation using only the Laplace transformation.

The rest of the paper is organized as follows. In Section 2, preliminaries and previous related works have been introduced. The existence and uniqueness results for linear problems have been presented in Section 3. Some simple but important initial and boundary value problems of the fraction Caputo-Fabrizio differential equation are given in Section 4. In Section 5, the existence and uniqueness of the nonlinear 
boundary value problem of the fraction Caputo-Fabrizio differential equation have been demonstrated. Finally, an application to a mass spring damper system is given in the last section.

\section{Preliminaries and Previous Results}

We present some definitions and previous results of the new fractional Caputo-Fabrizio derivative that are needed in this work.

Definition 2.1. Let $\alpha \in(0,1)$ and $f \in H^{1}(a, b), a<b$. The Caputo fractional derivative of the function $f$ defined as

$$
D_{C}^{\alpha} f(x)=\frac{1}{\Gamma(1-\alpha)} \int_{a}^{x}(x-t)^{\alpha} f^{\prime}(t) d t
$$

Definition 2.2. [14] Given $a<b$ and $f \in H^{1}(a, b)$, the fractional Caputo-Fabrizio derivative of the function of order $\alpha \in(0,1)$ is defined for $t \geq 0$

$$
{ }^{C F} D^{\alpha} f(x)=\frac{1}{1-\alpha} \int_{a}^{x} \exp \left(-\frac{\alpha}{1-\alpha}(x-t)\right) f^{\prime}(t) d t .
$$

Definition 2.3. [15] The Caputo-Fabrizio fractional integral of oder $\alpha \in(0,1)$ is defined as

$$
C F \mathscr{I}(f)(x)=(1-\alpha) f(x)+\alpha \int_{0}^{x} f(s) d s .
$$

The Caputo-Fabrizio fractional of order $\sigma=\alpha+n$ for $\alpha \in(0,1)$ and $n \in \mathbb{N}$ defined as

$$
{ }^{C F} D^{\alpha+n} f(x):={ }^{C F} D^{\alpha}\left({ }^{C F} D^{n} f(x)\right) .
$$

Theorem 2.4. [14] Let the function $f(x)$ satisfy $f^{(k)}(a)=0, \quad k=1,2, \ldots, n$, then the equality

$$
{ }^{C F} D^{\alpha}\left({ }^{C F} D^{n} f(x)\right)={ }^{C F} D^{n}\left({ }^{C F} D^{\alpha} f(x)\right)
$$

holds.

Definition 2.5. For $\sigma=\alpha+1$ with $\alpha \in(0,1)$, the Caupto-Fabrizio fractional derivative of order $\sigma$ defined as

$$
{ }^{C F} D^{\sigma} f(x)=\frac{1}{1-\alpha} \int_{a}^{x} \exp \left(-\frac{\alpha}{1-\alpha}(x-t)\right) f^{\prime \prime}(t) d t .
$$

Note that the equality ${ }^{C F} D^{\alpha}\left({ }^{C F} D^{1} f(x)\right)=C F D^{1}\left({ }^{C F} D^{\alpha} f(x)\right)$ is defined unambiguously when $f^{\prime}(0)=0$. (see [14])

Definition 2.6. For a function $f(x)$, the Laplace transformation $F(s)$ of $f$ is given by

$$
F(s)=\mathscr{L}[f(x)]=\int_{0}^{\infty} \exp (-s x) f(x) d x
$$

Lemma 2.7. [14] The Laplace transform of the Caputo-Fabrizio fractional of order $\sigma=\alpha+n$ for $\alpha \in(0,1)$ and $n \in \mathbb{N}$ is given by

$$
\mathscr{L}\left\{{ }^{C F} D^{\sigma}(f)(x)\right\}(s)=\frac{s^{n+1} \mathscr{L}\{f(x)\}(s)-s^{n} f(0)-s^{n-1} f^{\prime}(0)-\cdots-f^{(n)}(0)}{s+\alpha(1-s)} .
$$

\section{Existence and Uniqueness of the Solution}

We show the existence and uniqueness of the solution of the fractional differential equations involving the Caputo- Fabrizio fractional derivative in this section. We also derive the solution for some fractional differential equation that are important for physical applications.

Theorem 3.1. [15] For $\alpha \in(0,1)$ and $h \in L_{1}(0, \infty)$, the following first order fractional differential equation

$$
\begin{aligned}
& { }^{C F} D^{\alpha}(u)(x)=h(x), \quad x \geq 0 \\
& u(0)=u_{0}
\end{aligned}
$$

has the unique solution

$$
u(x)=u_{0}+(1-\alpha)(h(x)-h(0))+\alpha \int_{0}^{x} h(s) d s .
$$

Theorem 3.2. [15] For $\alpha \in(0,1)$ and $h \in L_{1}(0, \infty)$, the following first order fractional differential equation

$$
\begin{aligned}
& { }^{C F} D^{\alpha}(u)(x)=\lambda u(x)+h(x), \quad \lambda \neq 0, \quad x \geq 0 \\
& u(0)=u_{0}
\end{aligned}
$$

has the unique solution, when $\lambda(1-\alpha)=1$

$$
u(x)=-\frac{1-\alpha}{\lambda \alpha} u^{\prime}(x)-\frac{\alpha}{\lambda} u(x)
$$

and when $\lambda(1-\alpha) \neq 1$

$$
u(x)=\frac{\lambda \alpha}{1-\lambda(1-\alpha)} \int_{0}^{x} u(s) d s+u_{0}+\frac{1-\alpha}{1-\lambda(1-\alpha)}(h(x)-h(0))+\frac{\alpha}{1-\lambda(1-\alpha)} \int_{0}^{x} h(s) d s .
$$


Theorem 3.3. [15] If $\alpha \in(0,1)$, then the function $u$ solves the fractional differential equation

$$
{ }^{C F} D^{\alpha}(u)(x)=0, \quad x \geq 0
$$

if and only if $u$ is a constant function.

We study here the boundary value problem of a class of the Caputo-Fabrizio fractional differential equations of order $\sigma \in(1,2)$ on $[0,1]$

Theorem 3.4. For $\sigma=\alpha+1, \quad \alpha \in(0,1)$, and $g:[0, \infty) \rightarrow \mathbb{R}$ with $g \in L_{1}(0, \infty)$, the following boundary value problem of the fractional Caputo-Fabrizio differential equation

$$
\begin{aligned}
& { }^{C F} D^{\sigma}(u)(x)=g(x), \quad x \geq 0 \\
& u(0)=u_{0}, \quad u(1)=u_{1}
\end{aligned}
$$

has the unique solution given by

$$
\begin{aligned}
u(x) & =u_{0}+\left(u_{1}-u_{0}\right) x+(1-\alpha)(1-x) \int_{0}^{x} g(t) d t+\alpha(1-x) \int_{0}^{x} \operatorname{tg}(t) d t \\
& -(1-\alpha) x \int_{x}^{1} g(t) d t-\alpha x \int_{x}^{1}(1-t) g(t) d t .
\end{aligned}
$$

Proof. Applying the Laplace operator to the equation (3.1), we get

$$
\mathscr{L}\left\{{ }^{C F} D^{\sigma}(u)(x)\right\}(s)=\mathscr{L}\{g(x)\}(s)
$$

Appealing the Lemma 2.7, we are led to

$$
\frac{s^{2} U(s)-s u(0)-u^{\prime}(0)}{s+\alpha(1-s)}=G(s)
$$

where $U(s)=\mathscr{L}\{(u)(x)\}(s)$ and $G(s)=\mathscr{L}\{g(x)\}(s)$.

Equivalently, we can rewrite the last equation as

$$
U(s)=\frac{1}{s} u(0)+\frac{1}{s^{2}} u^{\prime}(0)+\frac{1-\alpha}{s} G(s)+\frac{\alpha}{s^{2}} G(s) .
$$

The inverse Laplace operator is applied to above equation to arrive at

$$
u(x)=u(0)+x u^{\prime}(0)+(1-\alpha) \int_{0}^{x} g(t) d t+\alpha \int_{0}^{x}(x-t) g(t) d t .
$$

Taking into account the boundary conditions (3.2), we have the desired result

$$
\begin{aligned}
u(x) & =u_{0}+\left(u_{1}-u_{0}\right) x+(1-\alpha)(1-x) \int_{0}^{x} g(t) d t+\alpha(1-x) \int_{0}^{x} t g(t) d t \\
& -(1-\alpha) x \int_{x}^{1} g(t) d t-\alpha x \int_{x}^{1}(1-t) g(t) d t .
\end{aligned}
$$

For the uniqueness, as usual, we suppose that there are two solutions of the problem, say $v_{1}$ and $v_{2}$. Then we must have

$$
{ }^{C F} D^{\sigma}\left(v_{1}\right)(x)-{ }^{C F} D^{\sigma}\left(v_{2}\right)(x)={ }^{C F} D^{\sigma}\left(v_{1}-v_{2}\right)(x)={ }^{C F} D^{\alpha}\left(D v_{1}-D v_{2}\right)(x)=0
$$

Thus, by Theorem 3.3 we get

$$
D v_{1}(x)=D v_{2}(x) \text {. }
$$

This implies that $v_{1}(x)=v_{2}(x)+c$ for some constant $c$. But the condition $v_{1}(0)=v_{2}(0)$ leads to $c=0$. That is $v_{1}(x)=v_{2}(x)$ for all $x \geq 0$.

Remark 3.5. In Theorem 3.4, if we let $h(x):=g(x)-g(0)$, then $h(0)=0$ so that the initial value problem

$$
\begin{aligned}
& { }^{C F} D^{\sigma}(u)(x)=h(x), \quad x \geq 0 \\
& u(0)=A, \quad u^{\prime}(0)=B
\end{aligned}
$$

has the unique solution of much simpler form given by

$$
u(x)=A+B x+(1-\alpha) \int_{0}^{x} h(t) d t+\alpha \int_{0}^{x}(x-t) h(t) d t .
$$

We further study the linear differential equation of fractional order in the sense of Caputo-Fabrizio fractional derivative, then we will work on nonlinear boundary value problems of the fractional Caputo-Fabrizio differential equations. We first give the results for the linear cases.

Theorem 3.6. If $\sigma \in(1,2)$ and $g \in L_{1}(0, \infty) \cap C^{1}[0, \infty)$, then the following linear boundary value problem of the fractional Caputo-Fabrizio differential equation has the unique solution for all $\eta \in \mathbb{R}$.

$$
\begin{aligned}
& { }^{C F} D^{\sigma}(u)(x)=\eta u(x)+g(x), \quad \eta \neq 0, \quad x \geq 0 \\
& u(0)=u_{0}, \quad u(1)=u_{1}
\end{aligned}
$$


Proof. The case when $\eta=0$ is already was proved in Theorem 3.4. So, assume that $\eta \neq 0$. we see that from Theorem 3.4, the solution to (3.4) and (3.5) can be written as

$$
\begin{aligned}
u(x) & =u_{0}+\left(u_{1}-u_{0}\right) x+(1-\alpha)(1-x) \int_{0}^{x}(\eta u(t)+g(t)) d t+\alpha(1-x) \int_{0}^{x} t(\eta u(t)+g(t)) d t \\
& -(1-\alpha) x \int_{x}^{1}(\eta u(t)+g(t)) d t-\alpha x \int_{x}^{1}(1-t)(\eta u(t)+g(t)) d t .
\end{aligned}
$$

A little algebraic manipulation reveals that

$$
\begin{aligned}
& u(x)+\eta x \int_{0}^{1}(1-\alpha t) u(t) d t-\eta \int_{0}^{x}(1-\alpha+x \alpha-t \alpha) u(t) d t \\
& =u_{0}+\left(u_{1}-u_{0}\right) x+(1-\alpha)(1-x) \int_{0}^{x} g(t) d t+\alpha(1-x) \int_{0}^{x} t g(t) d t \quad-(1-\alpha) x \int_{x}^{1} g(t) d t-\alpha x \int_{x}^{1}(1-t) g(t) d t
\end{aligned}
$$

Differentiating the equation (3.6) twice, we have that

$$
u^{\prime \prime}(x)-(1-\alpha) \eta u^{\prime}(x)=(1-\alpha) g^{\prime}(x)+\alpha g(x) .
$$

Now we have two cases to analyze. First, we assume that $(1-\alpha) \eta=0 \Leftrightarrow \alpha=1$ since $\eta \neq 0$. In this case, the equation (3.7) becomes

$$
u^{\prime \prime}(x)=g(x) .
$$

This is just a second order ordinary differential equation with solution given by

$$
u(x)=-u_{0} x+u_{0}+u_{1} x+(x-1) \int_{1}^{0}\left(\int_{1}^{s} g(y) d y\right) d s+\int_{1}^{x}\left(\int_{1}^{s} g(y) d y\right) d s .
$$

The second case when $(1-\alpha) \eta \neq 0$, we have

$$
\begin{aligned}
u(x) & =u_{0}+\int_{0}^{x} e^{(1-\alpha) \eta t} \int_{0}^{t} e^{(1-\alpha) \eta s}\left((1-\alpha) g^{\prime}(s)+\alpha g(s)\right) d s d t \\
& +\frac{u_{1}-u_{0}-\int_{0}^{1} e^{(1-\alpha) \eta t} \int_{0}^{t} e^{(1-\alpha) \eta s}\left((1-\alpha) g^{\prime}(s)+\alpha g(s)\right) d s d t}{\int_{0}^{1} e^{(1-\alpha) \eta t} d t} \int_{0}^{x} e^{(1-\alpha) \eta t} d t .
\end{aligned}
$$

\section{Solutions of the initial and boundary value problem of the linear Caputo-Fabrizio fractional differential equations}

In this section, some initial and boundary value problems of the fractional differential equation in the sense of the Caputo-Fabrizio derivative have been presented.

Example 4.1. If $\sigma=\alpha+1$ with $\alpha=\frac{1}{2}$ and $c_{1}, c_{2} \in \mathbb{R}$, then the following initial value problem of fractional differential equation

$$
\begin{aligned}
& u^{\prime \prime}(x)+c_{1}^{C F} D^{\sigma}(u)(x)=c_{2}^{C F} D^{\alpha} u(x)+1-\exp (-x) \\
& u(0)=0, \quad u^{\prime}(0)=0
\end{aligned}
$$

has a unique solution of the form

$$
u(x)=\frac{\exp \left(-\frac{3\left(2 c_{1}+1\right) x}{2}\right) \sinh \left(\sqrt{\frac{9 x\left(2 c_{1}+1\right)^{2}}{4}+2 c_{2}}\right)}{\sqrt{\frac{9 x\left(2 c_{1}+1\right)^{2}}{4}+2 c_{2}}} .
$$

In fact, by the Laplace transformation, the equation can be written as

$$
\begin{aligned}
& s^{2} U(s)-s u(0)-u^{\prime}(0)+c_{1} \frac{s^{2} U(s)-s u(0)-u^{\prime}(0)}{(s+1) / 2}-c_{2} \frac{s}{(s+1) / 2}=\frac{s}{s+1} \\
& U(s)\left(s^{3}+\left(2 c_{1}+1\right) s^{2}-2 c_{2} s\right)=s \\
& U(s)=\frac{s}{s^{3}+\left(2 c_{1}+1\right) s^{2}-2 c_{2} s}
\end{aligned}
$$

where $U(s)=\{\mathscr{L} u(t)\}(s)$. Now, the inverse Laplace transformation gives us that

$$
u(x)=\frac{\exp \left(-\frac{3\left(2 c_{1}+1\right) x}{2}\right) \sinh \left(\sqrt{\frac{9 x\left(2 c_{1}+1\right)^{2}}{4}+2 c_{2}}\right)}{\sqrt{\frac{9 x\left(2 c_{1}+1\right)^{2}}{4}+2 c_{2}}}
$$


Example 4.2. Consider the initial value problem

$$
\begin{aligned}
{ }^{C F} D^{\sigma} u(x)+u(x) & =0 \\
u(0)=1, \quad u^{\prime}(0) & =0
\end{aligned}
$$

where $\sigma=\alpha+1$ with $\alpha \in(0,1)$

Applying the Laplace transformation leads to have

$$
U(s)\left(s^{2}+s+\alpha(1-s)\right)=s .
$$

Now, the inverse Laplace transformation gives the exact solution as follows

$$
\begin{aligned}
& u(x)=\exp (x(\alpha / 2-1 / 2))\left(\cosh \left(x\left(\alpha^{2} / 4-3 \alpha / 2+1 / 4\right)^{1 / 2}\right)+\left(\operatorname { s i n h } \left(x \left(\alpha^{2} / 4\right.\right.\right.\right. \\
& \left.\left.\left.-3 \alpha / 2+1 / 4)^{1 / 2}\right)(\alpha / 2-1 / 2)\right) /\left(\alpha^{2} / 4-3 \alpha / 2+1 / 4\right)^{1 / 2}\right)
\end{aligned}
$$

Example 4.3. Consider the system of fractional algebraic-differential equations

$$
\begin{aligned}
& { }^{C F} D^{1 / 2} u(x)-x v(x)+u(x)-(1+x) v(t)=0 \\
& v(x)=\sin x \\
& u(0)=1, \quad v(0)=0
\end{aligned}
$$

Applying the Laplace transformation, one gets

$$
\begin{aligned}
& \frac{s U(s)-1}{(s+1) / 2}+V(s)+s V^{\prime}(s)+U(s)-V(s)+V^{\prime}(s)=0 \\
& V(s)=\frac{1}{s^{2}+1}, \quad V^{\prime}(s)=-\frac{2 s}{\left(s^{2}+1\right)^{2}} \\
& U(s)=\frac{s(s+1)}{\left(1+s^{2}\right)^{2}}+\frac{1}{s+1}
\end{aligned}
$$

Now, the inverse Laplace transform gives the exact solution

$$
u(x)=\frac{x+1}{2} \sin x+\frac{x}{2} \cos x+\exp (-x)
$$

Example 4.4. Consider the boundary value problem

$$
\begin{aligned}
& { }^{C F} D^{3 / 2} u(x)=\lambda u(x) \\
& u(0)=0, \quad u(1)=1
\end{aligned}
$$

This is the equation given in the problem (3.4) and (3.5) with $\sigma=1+1 / 2$ and $u_{0}=0, u_{1}=1$. Thus, the exact solution given by

$$
u(x)=\frac{1}{\int_{0}^{1} e^{(\lambda / 2) t} d t} \int_{0}^{x} e^{(\lambda / 2) t} d t=\frac{e^{\lambda x / 2}-1}{e^{\lambda / 2}-1}
$$

\section{Nonlinear boundary value problems}

We prove the existence and uniquness of the nonlinear boundary value problems of the Caputo-Fabrizio differential equations by the help of the Banach contraction principle.

Let $C(I)$ be the Banach space of continuous functions on $I=[0,1]$ with maximum norm

$$
\|x\|=\max _{s \in[0,1]}|x(s)|, \quad x \in C(I) .
$$

We now state the existence and uniquness of the solution in the next theorem.

Theorem 5.1. If $\sigma=1+\alpha, \quad \alpha \in(0,1]$ and $F:[0,1] \times \mathbb{R} \rightarrow \mathbb{R}$ is a continuous function with the property that

$$
\left|F\left(x, u_{1}\right)-F\left(x, u_{2}\right)\right| \leq q\left|u_{1}-u_{2}\right| \quad u_{1}, u_{2} \in \mathbb{R}, \quad q>0,
$$

then the boundary value problem

$$
\begin{aligned}
& { }^{C F} D^{\sigma}(u)(x)=F(x, u(x)), \quad x \geq 0 \\
& u(0)=u_{0}, \quad u(1)=u_{1}
\end{aligned}
$$

has a unique solution in $C(I)$ provided $q<1$. 
Proof. Let the operator $T: C(I) \rightarrow C(I)$ be given by

$$
\begin{aligned}
(T u)(x) & =u_{0}+\left(u_{1}-u_{0}\right) x+(1-\alpha)(1-x) \int_{0}^{x} F(t, u(t)) d t+\alpha(1-x) \int_{0}^{x} t F(t, u(t)) d t \\
& -(1-\alpha) x \int_{x}^{1} F(t, u(t)) d t-\alpha x \int_{x}^{1}(1-t) F(t, u(t)) d t
\end{aligned}
$$

We see that the solution for the problem (5.1) and (5.2) is the fixed point of the map $T$. For $u, v \in C(I)$ and $0 \leq t \leq 1$, we find that

$$
\begin{aligned}
|(T u)(x)-(T v)(x)| & =\mid(1-\alpha)(1-x) \int_{0}^{x}\left(F(t, u(t))-F(t, v(t)) d t+\alpha(1-x) \int_{0}^{x} t(F(t, u(t))-F(t, v(t)) d t\right. \\
& -(1-\alpha) x \int_{x}^{1}\left(F(t, u(t))-F(t, v(t)) d t-\alpha x \int_{x}^{1}(1-t)(F(t, u(t))-F(t, v(t)) d t \mid\right. \\
& \leq(1-\alpha)(1-x) x q\|u-v\|+\alpha(1-x) \frac{x^{2}}{2} q\|u-v\|+(1-\alpha) x(1-x) q\|u-v\|+\alpha x \frac{(1-x)^{2}}{2} q\|u-v\| \\
& =(1-x) x \frac{4-3 \alpha}{2} q\|u-v\| \leq \max _{x \in[0,1]}(1-x) x \frac{4-3 \alpha}{2} q\|u-v\| \leq \frac{4-3 \alpha}{8} q\|u-v\| \leq q\|u-v\| .
\end{aligned}
$$

Since $q<1$, the operator $T$ is a contraction, and by the Banach contraction theorem $T$ must have a unique fixed point that is the solution of the problem (5.1) and (5.2).

\section{An Application to a Mass-Spring-Damper System}

In [19], a mass spring damper system equation has been modelled by the Caputo-Fabrizio fractional differential equation as follows

$$
{\frac{m}{\mu^{2(1-\alpha)}}}^{C F} D^{2 \alpha} u(x)+{\frac{c}{\mu^{1-\alpha}}}^{C F} D^{\alpha} u(x)+k u(x)=F(x), \quad \alpha \in(0,1] .
$$

where $\mu$ is the dimension of second, $m$ is the damping coefficient, $c$ is the spring constant and $F(x)$ is the force of the system. The parameter $\mu$ is introduced because of the dimensionless quantity of the physical problem in the case of fractional derivative of the displacement. The equation (6.1) has been provided with an initial displacement, $u_{0}$, and velocity, $v_{0}=0$ for the mass $m$. As in [19], two cases for the forcing term will be considered. Additionally, the order of the fractional is also considered in two cases.

1 . Assume that the forcing term $F(x)=A$ for some constant $A$. Moreover, suppose that $\alpha \in(0,1 / 2)$ so that $2 \alpha \in(0,1)$. Applying the Laplace transform of (6.1) leads to get

$$
\begin{aligned}
& \frac{s U(s)-u_{0}}{s+2 \alpha(1-s)}+c \frac{\eta^{2} \mu^{\alpha-1}}{k}\left(\frac{s U(s)-u_{0}}{s+\alpha(1-s)}\right)+\eta^{2} U(s)=A \frac{\eta^{2}}{k} \frac{1}{s} \\
& U(s)=\frac{u_{0}(s+\alpha(1-s)+B(s+2 \alpha(1-s)))}{s\left(s+\alpha(1-s)+s+2 \alpha(1-s)\left(B+\eta^{2}(s+\alpha(1-s))\right)\right.} \\
& +\frac{\left(\eta^{2} / k\right) A(s+2 \alpha(1-s))(s+\alpha(1-s))}{s^{2}\left(s+\alpha(1-s)+s+2 \alpha(1-s)\left(B+\eta^{2}(s+\alpha(1-s))\right)\right.}
\end{aligned}
$$

where $\frac{\eta^{2}}{k}=\frac{\mu^{2(1-\alpha)}}{m}$ and $B=c \frac{\eta^{2}}{k} \mu^{\alpha-1}$

The inverse Laplace transform yields the exact solution

$$
\begin{aligned}
& u(x)=\left(A \eta^{2} / k\right)\left(\frac{2 \alpha x}{\left(2 \alpha \eta^{2}+2 B+1\right.}-\frac{-2 \alpha \eta^{2}+2 \alpha-6 B+4 \alpha B+1}{\left(2 \alpha \eta^{2}+2 B+1\right)^{2}} \exp \left(\frac{x\left(\alpha+2 \alpha B-2 \alpha \eta^{2}+4 \alpha^{2} \eta^{2}-2\right)}{4 \alpha \eta^{2}(\alpha-1)}\right.\right. \\
& \left(\cosh \left(\left(x\left(4 \alpha^{2} B^{2}+8 \alpha^{2} B \eta^{2}+4 \alpha^{2} B+4 \alpha^{2} n^{4}-12 \alpha^{2} \eta^{2}+\alpha^{2}-8 \alpha B+8 \alpha \eta^{2}-4 \alpha+4\right)^{1 / 2}\right) /\left(4 \alpha \eta^{2}(\alpha-1)\right)\right)+\right. \\
& \left(\sinh \left(\left(x\left(4 \alpha^{2} B^{2}+8 \alpha^{2} B \eta^{2}+4 \alpha^{2} B+4 \alpha^{2} n^{4}-12 \alpha^{2} \eta^{2}+\alpha^{2}-8 \alpha B+8 \alpha \eta^{2}-4 \alpha+4\right)^{1 / 2}\right) /\left(4 \alpha \eta^{2}(\alpha-1)\right)\right)\right. \\
& \left.\left(8 \alpha^{2} B^{2}+4 \alpha^{2} B \eta^{2}+8 \alpha^{2} B+4 \alpha^{2} n^{4}-14 \alpha^{2} \eta^{2}+2 \alpha^{2}-12 \alpha B^{2}+4 \alpha B \eta^{2}-12 \alpha B+6 \alpha \eta^{2}-3 \alpha+8 B^{2}-4 B+4\right)\right) \\
& \left.\left.\div\left(\left(-2 \alpha \eta^{2}+2 \alpha-6 B+4 \alpha B+1\right)\left(4 \alpha^{2} B^{2}+8 \alpha^{2} B \eta^{2}+4 \alpha^{2} B+4 \alpha^{2} n^{4}-12 \alpha^{2} \eta^{2}+\alpha^{2}-8 \alpha B+8 \alpha \eta^{2}-4 \alpha+4\right)^{(} 1 / 2\right)\right)\right) \\
& \left.\left.\left.\left(-2 \alpha \eta^{2}+2 \alpha-6 B+4 \alpha B+1\right)\right) /\left(2 \alpha \eta^{2}+2 B+1\right)^{2}\right)\right) \\
& +(2 B+1) /\left(2 \alpha n^{2}+2 B+1\right)-\left(e x p\left(\left(x\left(\alpha+2 \alpha B-2 \alpha \eta^{2}+4 \alpha^{2} \eta^{2}-2\right)\right) /\left(4 \alpha \eta^{2}(\alpha-1)\right)\right)(2 B+1)\right. \\
& \left(\cosh \left(\left(x\left(4 \alpha^{2} B^{2}+8 \alpha^{2} B \eta^{2}+4 \alpha^{2} B+4 \alpha^{2} \eta^{4}-12 \alpha^{2} \eta^{2}+\alpha^{2}-8 \alpha B+8 \alpha \eta^{2}-4 \alpha+4\right)^{1 / 2}\right) /\left(4 \alpha \eta^{2}(\alpha-1)\right)\right)+\right. \\
& \left(\sinh \left(\left(x\left(4 \alpha^{2} B^{2}+8 \alpha^{2} B \eta^{2}+4 \alpha^{2} B+4 \alpha^{2} \eta^{4}-12 \alpha^{2} \eta^{2}+\alpha^{2}-8 \alpha B+8 \alpha \eta^{2}-4 \alpha+4\right)^{1 / 2}\right) /\left(4 \alpha \eta^{2}(\alpha-1)\right)\right)\right. \\
& \left.\left(\alpha-2 B+4 \alpha B+4 \alpha B^{2}-2 \alpha \eta^{2}-4 B^{2}\right)\right) /((2 B+1) \\
& \left.\left.\left.\left(4 \alpha^{2} B^{2}+8 \alpha^{2} B \eta^{2}+4 \alpha^{2} B+4 \alpha^{2} \eta^{4}-12 \alpha^{2} \eta^{2}+\alpha^{2}-8 \alpha B+8 \alpha \eta^{2}-4 \alpha+4\right)^{1 / 2}\right)\right)\right) /\left(2 \alpha \eta^{2}+2 B+1\right)
\end{aligned}
$$


2 . Assume that the forcing term $F(x)=A$ for some constant $A$. Moreover, suppose that $\alpha \in(1 / 2,1)$ so that $2 \alpha \in(1,2)$. Applying the Laplace transform of (6.1) leads to get

$$
\begin{aligned}
& \frac{s^{2} U(s)-u_{0}}{s+\alpha(1-s)}+c \frac{\eta^{2} \mu^{\alpha-1}}{k}\left(\frac{s U(s)-u_{0}}{s+\alpha(1-s)}\right)+\eta^{2} U(s)=A \frac{\eta^{2}}{k} \frac{1}{s} \\
& U(s)=\frac{u_{0}(s+\alpha(1-s)+B(s+2 \alpha(1-s)))}{s\left(s+\alpha(1-s)+s+2 \alpha(1-s)\left(B+\eta^{2}(s+\alpha(1-s))\right)\right.} \\
& +\frac{\left(\eta^{2} / k\right) A(s+2 \alpha(1-s))(s+\alpha(1-s))}{s^{2}\left(s+\alpha(1-s)+s+2 \alpha(1-s)\left(B+\eta^{2}(s+\alpha(1-s))\right)\right.}
\end{aligned}
$$

where $\frac{\eta^{2}}{k}=\frac{\mu^{2(1-\alpha)}}{m}$ and $B=c \frac{\eta^{2}}{k} \mu^{\alpha-1}$

The inverse Laplace transform yields

$$
\begin{aligned}
& u(x)=u_{0}\left(\left(\exp \left(-x\left(B / 2-\left(\alpha \eta^{2}\right) / 2+\eta^{2} / 2\right)\right) \sinh \left(x\left(\left(\alpha^{2} \eta^{4}\right) / 4-\left(\alpha B \eta^{2}\right) / 2-\left(\alpha \eta^{4}\right) / 2-\alpha \eta^{2}+B^{2} / 4+\left(B \eta^{2}\right) / 2+\eta^{4} / 4\right)^{1 / 2}\right)\right.\right. \\
& \left.(B+1)) /\left(\left(\alpha^{2} \eta^{4}\right) / 4-\left(\alpha B \eta^{2}\right) / 2-\left(\alpha \eta^{4}\right) / 2-\alpha \eta^{2}+B^{2} / 4+\left(B \eta^{2}\right) / 2+\eta^{4} / 4\right)^{1 / 2}\right) \\
& +\left(A \eta^{2} / k\right)\left(1 / \eta^{2}-\left(\operatorname { e x p } ( - x ( B / 2 - ( \alpha \eta ^ { 2 } ) / 2 + \eta ^ { 2 } / 2 ) ) \left(\operatorname { c o s h } \left(x \left(\left(\alpha^{2} \eta^{4}\right) / 4-\left(\alpha B \eta^{2}\right) / 2-\left(\alpha \eta^{4}\right) / 2-\alpha \eta^{2}+B^{2} / 4+\left(B \eta^{2}\right) / 2\right.\right.\right.\right.\right. \\
& \left.\left.+\eta^{4} / 4\right)^{1 / 2}\right)+\left(\sinh \left(x\left(\left(\alpha^{2} \eta^{4}\right) / 4-\left(\alpha B \eta^{2}\right) / 2-\left(\alpha \eta^{4}\right) / 2-\alpha \eta^{2}+B^{2} / 4+\left(B \eta^{2}\right) / 2+\eta^{4} / 4\right)^{1 / 2}\right)\left(B / 2+\left(\alpha \eta^{2}\right) / 2-\eta^{2} / 2\right)\right) \\
& \left.\left.\left.\div\left(\left(\alpha^{2} \eta^{4}\right) / 4-\left(\alpha B \eta^{2}\right) / 2-\left(\alpha \eta^{4}\right) / 2-\alpha \eta^{2}+B^{2} / 4+\left(B \eta^{2}\right) / 2+\eta^{4} / 4\right)^{1 / 2}\right)\right) / \eta^{2}\right)
\end{aligned}
$$

\section{References}

[1] KS. Miller, B. Ross, (Eds.), An Introduction to the Fractional Calculus and Fractional Differential Equations, John Wiley, NY 1993.

[2] I. Podlubny, Fractional Differential Equations, Academic Press, New York 1999.

[3] A.A. Kilbas, H. M. Srivastava and J. J. Trujillo, Theory and Applications of Fractional Differential Equations, North-Holland Mathematical Studies, Amsterdam, 2006.

[4] J. Sabatier, P. Lanusse, P. Melchior, A. Oustaloup,Fractional Order Differentiation and Robust Control Design, Springer, 2015.

[5] F. Mainardi, Fractional Calculus and Waves in linear Viscoelasticity: an Introduction to Mathematical Models, World Scientific, 2010.

[6] V.V. Uchaikin, Fractional Derivatives for Physicists and Engineers, Springer, 2013.

[7] R. J. Greechie, S. P. Gudder, The Fractional Calculus Theory and Applications of Differentiation and Integration to Arbitrary Order, 111 Elsevier, 1974

[8] A. N. Kochubei, Fractional Differential Equations: an Introduction to Fractional Derivatives, Fractional Differential equations, to Methods of Their Solution and some of their Applications, 198 Academic Press 1998.

[9] F. Usta, Fractional Type Poisson Equations by Radial Basis Functions Kansa Approach, J. Inequal. Spec. Funct., (7)4, (2016),143-149.

[10] M. Z. Sarikaya and F. Usta, On Comparison Theorems for Conformable Fractional Differential Equations, Int. J. Anal. Appl., (12)2, (2016), $207-214$.

[11] F. Usta, A mesh-free technique of numerical solution of newly defined conformable differential equations, Konuralp J. Math., (4)2,(2016) $149-157$.

[12] F. Usta and M. Z. Sarkaya, The analytical solution of Van der Pol and Lienard differential equations within conformable fractional operator by retarded integral inequalities, Demonstr. Math., 52(1), (2019), 204-212.

[13] Fuat Usta, Computational solution of Katugampola conformable fractional differential equations via RBF collocation method, AIP Conference Proceedings, 1833(1) (2017), 200461-200464., Doi: http://dx.doi.org/10.1063/1.4981694.

[14] M. Caputo, M. Fabrizio, A New Definition of Fractional Derivative without Singular Kerne, Progr. Fract. Differ. Appl., 1:1 (2015), 1-13.

[15] J. Losada, J.J. Nieto, Properties of a new fractional derivative without singular kernel, Progr. Fract. Differ. Appl., 1 (2016), 87-92.

[16] M. Caputo, M. Fabrizio, Applications of new time and spatial frac-tional derivatives with exponential kernels, Progr. Fract. Differ. Appl., 2 (2016), 1-11.

[17] Xiao-Jun Yang, H.M.Srivastava, J.A.Machado Tenreiro, A new fractional derivative without singular kernel, Thermal Science, (2015), doi:10.2298/TSCI151224222Y.

[18] Xiao-Jun Yang, H.M.Srivastava, J.A.Machado Tenreiro, Modeling diffusive transport with a fractional derivative without singular kernel, Physic A, 447 (2016), 467-481.

[19] J.F.G. Aguilar, H. Y. Martinez, C.C. Ramon, I.C. Ordunia, R.F. E. Jimenez, V.H.O. Peregrino, Modeling of a Mass-Spring-Damper System by Fractional Derivatives with and without a Singular Kernel, Entropy, 17 (2015), 6289-6303.

[20] M.Yavuz, N. Özdemir, European Vanilla Option Pricing Model of Fractional Order without Singular Kernel , Fractal and Fractional, (2)1, 3 (2018).

[21] F. Evirgen, M.Yavuz, An alternative approach for nonlinear optimization problem with Caputo-Fabrizio derivative , In ITM Web of Conferences , 22, EDP Sciences, (2018),p. 01009

[22] M.Yavuz, N. Özdemir, Comparing the new fractional derivative operators involving exponential and Mittag Leffler kernel, Discrete Contin. Dyn. Syst., 13(3), (2019), 1098-1107.

[23] D. Zhao, M. Luo, Representations of acting processes and memory effects: General fractional derivative and its application to theory of heat conduction with finite wave speeds, Appl. Math. Comput., 346 (2019), 531-544. 